\title{
Repercussões clínicas da exposição à poluição atmosférica*
}

\author{
Clinical repercussions of exposure to atmospheric pollution
}

JOSÉ EDUARDO DELFINI CANÇADO', ALFESIO BRAGA', LUIZ ALBERTO AMADOR PEREIRA', MARCOS ABDO ARBEX' ${ }^{1}$, PAULO HILÁRIO NASCIMENTO SALDIVA ${ }^{2}$, UBIRATAN DE PAULA SANTOS ${ }^{3}$

\begin{abstract}
RESUMO
A poluição atmosférica é um assunto exaustivamente discutido mundialmente. As repercussões clínicas decorrentes das exposições aos principais poluentes atmosféricos são apresentadas resumidamente. Os padrões de qualidade do ar para esses agentes, segundo a Agência de Proteção Ambiental Americana, estão descritos em relação aos seus valores primários e tempo médio. Os efeitos respiratórios conseqüentes à queima de combustíveis fósseis e de biomassa são apresentados de forma sucinta visando, essencialmente, a alertar os profissionais da saúde para o aumento da morbidade relacionada com a poluição ambiental.
\end{abstract}

Descritores: Doenças respiratórias/epidemiologia; Exposição ambiental; Doenças ocupacionais; Poluição ambiental; Saúde ambiental; Poluentes do ar; Saúde ocupacional

\begin{abstract}
Atmospheric pollution is a topic of extensive discussion the world over. The clinical repercussions of exposure to the principal atmospheric pollutants are summarized herein. According to the American Environmental Protection Agency, air quality standards for these agents are set based on their primary and half-life values. The respiratory effects of the burning of fossil fuels and biomass are succinctly presented, with a special focus on alerting health care professionals of the increased morbidity related to environmental pollution.
\end{abstract}

Descritores: Respiratory tract diseases/epidemiology; Environmental exposure; Occupational diseases; Environmental pollution; Environmental health; Air pollutants; Occupational health

\section{INTRODUÇÃO}

A poluição atmosférica pode ser definida como a presença de substâncias estranhas na atmosfera, resultantes da atividade humana ou de processos naturais, em concentrações suficientes para interferir direta ou indiretamente na saúde, segurança e bem estar dos seres vivos. ${ }^{(1)}$

As fontes naturais de poluição do ar são a queima acidental de biomassa (qualquer material derivado de plantas ou animais) e erupções vulcâni- cas, as quais podem ser consideradas as mais antigas fontes de contaminação do ar.

A queima de biomassa, em ambientes externos e internos, utilizada desde a pré-história para produção de energia, tem sido uma das importantes fontes antropogênicas de poluição atmosférica. A partir da Revolução Industrial, surgiram novas fontes de poluição do ar devido à queima de combustíveis fósseis nos motores a combustão e nas

\footnotetext{
* Trabalho realizado no Laboratório de Poluição Atmosférica do Departamento de Patologia da Faculadde de Medicina da Universidade de São Paulo - FMUSP - São Paulo (SP) Brsil.

1. Dutor em Ciência pela Faculadde de Medicina da Universidade de São Paulo - FMUSP - São Paulo (SP) Brsil.

2. Professor Titular do Departamento de Patologia da Faculdade de Medicina da Universidade de São Paulo - FMUSP.

3. 2. Médico Assistente Doutor da Disciplina de Pneumologia do Instituto do Coração do Hospital das Clínicas da Faculdade de Medicina da Universidade de São Paulo - InCor-HCFMUSP - São Paulo (SP) Brasil.

Endereço para correspondência: José Eduardo Delfini Cançado. Av. Dr. Arnaldo, 455, 10 andar, Sala 108, Cerqueira César, CEP: 01246-903. São Paulo, SP, Brasil. Tel 5511 3061-2789. E-mail: jose.cancado@merconet.com.br
} 
indústrias siderúrgicas e, mais recentemente, nos veículos automotivos, além dos produtos químicos. Estes processos não foram acompanhados de análises que pudessem avaliar seu impacto sobre o meio ambiente, a toxicidade dos resíduos produzidos e os prováveis danos à saúde.

Como conseqüência, no início do século XX ocorreram três episódios de elevações abruptas da concentração de poluentes do ar ocasionando aumentos da morbimortalidade (Vale do Meuse França, ${ }^{(2)}$ Donora - Pensilvânia - EUA, ${ }^{(3)}$ e Londres - Inglaterra ${ }^{(4)}$. Esses episódios estimularam a realização de diversos estudos epidemiológicos e experimentais, que identificaram os principais poluentes e suas repercussões sobre a saúde. Baseados nos achados decorrentes, vários países estabeleceram padrões de qualidade, ou seja, os limites máximos tolerados, a partir dos quais, a população exposta sofreria danos à saúde (Quadro 1).

No Brasil, em 1990, o Conselho Nacional de Meio Ambiente adotou os mesmos padrões. Vale ressaltar que esses não são os únicos, mas os principais poluentes atmosféricos. Posteriormente, novos estudos mostraram que não existem níveis seguros de concentração de poluentes para a saúde humana, questionando a segurança dos padrões de qualidade do ar estabelecidos. ${ }^{(5-7)}$

A seguir fazemos uma breve revisão dos principais poluentes atmosféricos.

\section{MATERIAL PARTICULADO}

0 material particulado é uma mistura de partículas líquidas e sólidas em suspensão no ar. Sua composição e tamanho dependem das fontes de emissão. As partículas podem ser divididas em dois grupos: partículas grandes (coarse mode), com diâmetro entre 2,5 e $30 \mathrm{~m}$, emitidas através de combustões descontroladas, dispersão mecânica do solo ou outros materiais da crosta terrestre (polens, esporos e materiais biológicos também se encontram nesta faixa de tamanho); e partículas pequenas (fine mode), com diâmetro menor que 2,5 m, emitidas pela combustão de fontes móveis e estacionárias, como automóveis, incineradores e termoelétricas, que por serem de menor tamanho e mais ácidas, podem atingir as porções mais inferiores do trato respiratório.

É oportuno salientar que a determinação da Agência de Proteção Ambiental Americana para controle de partículas menores ou iguais a $10 \mathrm{~m}$ (PM10), também chamadas de partículas inaláveis, se baseou no fato de que estas são as partículas que podem atingir as vias respiratórias inferiores, e não na sua composição química. Este material particulado inalável apresenta a importante característica de transportar gases adsorvidos em sua superfície, transportando-os até as porções mais distais das vias aéreas, onde ocorrem as trocas de gases no pulmão.

Um estudo realizado com monitores pessoais para $\mathrm{PM}_{10}$ e monitores colocados dos lados externo e interno das residências mapeou a composição do material particulado domiciliar. Aproximadamente 50\% do material particulado no interior das casas é proveniente do ambiente externo. 0 restante tem origem na combustão de tabaco, no fogão a gás ou indeterminada.

À medida que vão se depositando no trato respiratório, essas partículas passam a ser removidas pelos mecanismos de defesa. 0 primeiro deles é o espirro, desencadeado por grandes partículas que, devido ao seu tamanho, não conseguem ir além das narinas, onde acabam se depositando. Outros

Quadro 1 - Padrões de qualidade do ar para os principais poluentes segundo a Environmental Protection Agency - EPA, EUA

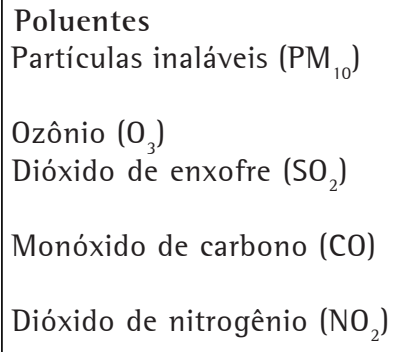

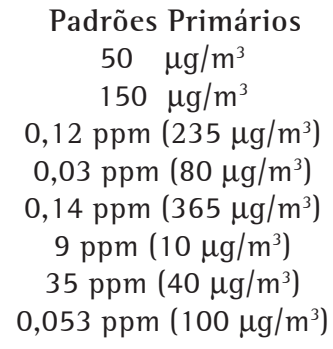

\section{Tempo Médio} média aritmética anual nível limite para 24 horas média de 1 hora máxima diária média aritmética anual nível máximo em 24 horas média máxima de 8 horas nível máximo em 1 hora média aritmética anual 
importantes mecanismos de defesa são a tosse e o aparelho mucociliar. Aquelas partículas que atingem as porções mais distais das vias aéreas são fagocitadas pelos macrófagos alveolares, sendo então removidas via aparelho mucociliar ou sistema linfático.

\section{MATERIAL GASOSO}

\section{Ozônio $\left(\mathrm{O}_{3}\right)$}

0 ozônio presente na troposfera, a porção da atmosfera em contato com a crosta terrestre, é formado por uma série de reações catalisadas pela luz do sol (raios ultravioleta) envolvendo, como precursores, óxidos de nitrogênio (NOx) e hidrocarbonetos, derivados das emissões de veículos, indústrias e usinas termoelétricas. Outras fontes de produção de ozônio são os purificadores de ar e máquinas de fotocópias.

0 ozônio é um potente oxidante e citotóxico, atingindo as porções mais distais das vias aéreas.

Dióxido de enxofre $\left(\mathrm{SO}_{2}\right)$ e aerossóis ácidos

Resultantes da combustão de elementos fósseis, como carvão e petróleo, têm como fontes principais os automóveis e termoelétricas. Uma vez lançado na atmosfera, o dióxido de enxofre pode ser transportado para regiões distantes das fontes primárias de emissão, o que aumenta sua área de atuação.

A maior parte do dióxido de enxofre inalado por uma pessoa em repouso é absorvida nas vias aéreas superiores. A atividade física leva a um aumento da ventilação alveolar, com conseqüente aumento da sua absorção pelas regiões mais distais do pulmão.

Os aerossóis ácidos mais comuns são o sulfato $\left(\mathrm{SO}_{4}^{--}\right)$, o bissulfato $\left(\mathrm{HSO}_{4}^{-}\right)$e o ácido sulfúrico $\left(\mathrm{H}_{2} \mathrm{SO}_{4}\right)$, encontrados dissolvidos nas gotas de água presentes na atmosfera. Os aerossóis ácidos causam inflamação do trato respiratório por apresentarem $\mathrm{pH}<1$.

\section{Monóxido de carbono (CO)}

As principais fontes emissoras de monóxido de carbono são os veículos automotivos, aquecedores a óleo, queima de tabaco, churrasqueiras e fogões a gás. 0 monóxido de carbono apresenta afinidade pela hemoglobina 240 vezes maior que a do oxigênio, o que faz com que uma pequena quantidade de monóxido de carbono possa saturar uma grande quantidade de moléculas de hemoglobina. Como conseqüência, ocorre diminuição da capacidade do sangue de transportar oxigênio e desvio da curva de dissociação da hemoglobina para a esquerda, o que pode causar hipóxia tecidual.

\section{Óxidos de nitrogênio (NOX)}

As principais fontes de óxido nítrico (NO) e dióxido de nitrogênio $\left(\mathrm{NO}_{2}\right.$ ) são os motores dos automóveis e, em menor escala, as usinas termoelétricas, indústrias, fogões a gás, aquecedores que utilizam querosene (mais freqüentes em regiões frias) e o cigarro. 0 dióxido de nitrogênio, na presença de luz solar, reage com hidrocarbonetos e oxigênio formando ozônio, sendo um dos principais precursores deste poluente na troposfera. 0 dióxido de nitrogênio, quando inalado, atinge as porções mais periféricas do pulmão devido à sua baixa solubilidade em água. Seu efeito tóxico está relacionado ao fato de ele ser um agente oxidante.

\section{EFEITOS RESPIRATÓRIOS DA POLUIÇÃO DO AR}

A poluição do ar causa uma resposta inflamatória no aparelho respiratório induzida pela ação de substâncias oxidantes, as quais acarretam aumento da produção, da acidez, da viscosidade e da consistência do muco produzido pelas vias aéreas, levando, conseqüentemente, à diminuição da resposta e/ou eficácia do sistema mucociliar. ${ }^{(5)}$

Efeitos respiratórios agudos da poluição do ar pela queima de combustíveis fósseis

A literatura biomédica está repleta de estudos sobre os efeitos agudos da poluição do ar sobre a saúde. ${ }^{(8)}$ A maior ênfase é dada a dois desfechos: mortalidade e admissões hospitalares.

A associação entre mortalidade e admissões hospitalares por doenças respiratórias e exposição à poluição do ar tem sido investigada, de forma mais sistemática, desde o começo da década de 1990. ${ }^{(9)} 0$ projeto APHEA (Air Pollution and Health: a European Approach) tem utilizado dados coletados em 29 cidades européias. Na sua fase dois, este projeto analisou as internações hospitalares por asma e doença pulmonar obstrutiva crônica entre indivíduos com mais de 65 anos e encontrou um aumento de 1\% (intervalo de confi- 
ança 1C95\%: 0,4 - 1,5) nas internações por doença pulmonar obstrutiva crônica para um aumento de $10 \mathrm{~g} / \mathrm{m}^{3}$ na concentração de PM10.(10)

Nos EUA, o National Mortality, Morbidity and Air Pollution Studies (NMMAPS) utilizou dados das vinte maiores regiões metropolitanas, com um total de 50 milhões de habitantes, entre 1987 e 1994. Em um estudo sobre admissões hospitalares em dez cidades, para todas as idades, foi encontrado um aumento de 1,5\% (1C95\%: 1,0 - 1,9) nas internações por doença pulmonar obstrutiva crônica para aumentos de $10 \mathrm{~g} / \mathrm{m}^{3}$ do $\mathrm{PM}_{10}{ }^{\left({ }^{(11)}\right.}$ Nessas mesmas cidades, para a mesma variação de $\mathrm{PM}_{10}$, observou-se aumento de mortes por pneumonia $(2,7 \%$; 1C95\%: $1,5-3,9)$ e por doença pulmonar obstrutiva crônica (1,7\%; 1C95\%:0,1 - 3,3), em uma cadeia lógica de efeitos mórbidos, dos menos graves, internações hospitalares, até os mais graves, mortes. ${ }^{(12)}$

No Brasil, no início da década de 1990, estudos associaram o dióxido de nitrogênio com mortalidade por doenças respiratórias em crianças ${ }^{(6)} \mathrm{e}$ o $\mathrm{PM}_{10}$ com a mortalidade em adultos. ${ }^{(13)}$ Estudos complementares confirmaram que as crianças são muito susceptíveis e relacionaram aumentos nos atendimentos de pronto-socorros ${ }^{(14)} \mathrm{e}$ de internações hospitalares ${ }^{(15-16)}$ com aumentos de $\mathrm{PM}_{10}$, dióxido de enxofre e monóxido de carbono, até cinco dias após a elevação do poluente.

Aumentos nas internações por doenças respiratórias crônicas de $18 \%$ e $14 \%$, respectivamente para doença pulmonar obstrutiva crônica e asma, em idosos, foram associados a variações diárias nas concentrações de dióxido de enxofre $(11,82$ $\left.\mu \mathrm{g} / \mathrm{m}^{3}\right)$ e ozônio $\left(35,87 \mu \mathrm{g} / \mathrm{m}^{3}\right) .{ }^{(17)}$

Em resumo, os efeitos agudos da poluição do ar sobre as doenças respiratórias estão associados a diferentes poluentes e afetam, predominantemente, as crianças e os idosos.

Efeitos respiratórios crônicos da poluição do ar pela queima de combustiveis fósseis

Estudos de coorte têm abordado os efeitos crônicos da poluição do ar, produzida pela queima de combustíveis fósseis, na mortalidade e na morbidade. Além deles, estudos transversais têm sido realizados, assumindo-se que exposições atuais podem representar de forma adequada exposições crônicas, e relacionando-as com as condições de saúde atuais da população. Em um estudo de painel realizado em oito comunidades suiças, a função pulmonar em adultos foi inversamente associada com elevações nas concentrações de $\mathrm{PM}_{10}$, dióxido de nitrogênio e dióxido de enxofre. ${ }^{(18)}$ Em 24 comunidades localizadas no Canadá e nos EUA foram encontradas associações significativas entre exposição a partículas finas e redução da função pulmonar com sintomas de bronquite, em crianças. ${ }^{(19)}$

Não tem sido possível relacionar variações na prevalência de asma com poluição do ar. ${ }^{(20)}$ Entretanto, estudos prospectivos realizados na Califórnia (EUA) relatam a associação entre o ozônio e incidência de asma. ${ }^{(21-22)} 0$ mesmo efeito não foi observado para outros poluentes.

Um estudo de coorte realizado nos EUA mostrou que exposição crônica ao material particulado fino aumenta o risco de doenças cardíacas e respiratórias, inclusive, de câncer de pulmão. ${ }^{(23)}$

Em um estudo de coorte realizado na Europa, encontrou-se uma associação entre mortalidade por doenças respiratórias e cardiovasculares com o local de moradia. Quanto mais próxima a residência de vias de grande fluxo de veículos, maior o risco de morrer por essas doenças (risco relativo de 1,95; 1C95\%: 1,09-3,52). ${ }^{(24)}$

0 Quadro 2 mostra os principais efeitos respiratórios adversos associados aos poluentes do ar originados da queima de combustíveis fósseis. ${ }^{(25)}$

Efeitos respiratórios da poluição do ar pela queima de biomassa

Recentemente foi publicada uma revisão sobre os efeitos da queima de biomassa na saúde da população exposta. ${ }^{(26)}$ Estudos epidemiológicos anteriores em comunidades expostas à fumaça proveniente da queima de biomassa em ambientes internos já indicavam uma relação consistente entre a exposição e o desenvolvimento de doença pulmonar crônica em adultos, ${ }^{(27)}$ além de bronquiectasias e fibrose pulmonar ${ }^{(28)} \mathrm{e}$ infecções respiratórias em crianças, ${ }^{(29)}$ provavelmente devido às alterações no mecanismo mucociliar, e à redução do poder de fagocitose dos macrófagos pulmonares. ${ }^{(30)}$ Outros estudos confirmaram que a exposição à queima de biomassa em ambientes internos está associada ao aumento da incidência de asma em adultos e crianças, ${ }^{(31)}$ diminuição da função pulmonar em crianças $^{(32)}$ e maior incidência de tuberculose pulmonar ${ }^{(33)}$ e de recém-nascidos de baixo peso. ${ }^{(34)}$

A queima de biomassa em ambientes abertos 
Quadro 2 - Principais efeitos respiratórios adversos associados aos poluentes do ar originados da queima de combustíveis fósseis
A. Aumento da mortalidade;
B. Aumento da incidência de câncer de pulmão;
C. Aumento da freqüência dos sintomas e das crises de asma;
D. Aumento da incidência de infecções respiratórias baixas;
E. Aumento das exacerbações em indivíduos já portadores de doenças cardiorrespiratórias ou outras
1. Redução da habilidade de exercer as tarefas diárias (geralmente por piora da dispnéia ou da angina pectoris);
2. Aumento das hospitalizações, tanto na freqüência como na duração;
3. Aumento das visitas médicas e à emergência;
4. Aumento do uso de medicamentos;
F. Redução do VEF, ou CVF associada a sintomas clínicos e ao aumento da mortalidade;
G. Aumento da prevalência de chiado;
H. Aumento da prevalência ou incidência de aperto no peito;
1. Aumento da prevalência ou incidência de tosse e hipersecreção pulmonar;
J. Aumento da incidência de infecções de vias aéreas superiores piorando a qualidade de vida;
K. Irritação nos olhos, garganta e narinas podendo interferir na vida normal.

$\mathrm{VEF}_{1}$ : volume expiratório forçado no primeiro segundo; CVF: capacidade vital forçada.

também produz poluição atmosférica e causa impacto sobre a saúde de indivíduos expostos.

Em 1997, ocorreram 1.500 focos de incêndios que queimaram 4,5 milhões de hectares de florestas na Indonésia, e foi observado um aumento nos atendimentos por asma, bronquite crônica e infecções respiratórias agudas. ${ }^{(35)}$ Alguns autores compararam a contagem de leucócitos no sangue periférico de 30 voluntários sadios (militares), no período das queimadas e após ele, e observaram um aumento significativo, às custas de polimorfonucleares, o que sugeriu ser esta uma resposta que pode contribuir para a patogênese da morbidade cardiorrespiratória associada a episódios agudos de poluição do ar. ${ }^{(36)}$

No Brasil, são plantados 5.000.000 de hectares de cana-de-açúcar e, por razões de produtividade e de segurança, sua colheita é realizada após a queima dos canaviais. Esta queima gera grande quantidade de material particulado expondo milhões de pessoas, durante quase seis meses, todos os anos, a este tipo de poluição atmosférica.

A gravidade desta situação fez com que o Laboratório de Poluição Atmosférica Experimental do Departamento de Patologia da Faculdade de Medicina da Universidade de São Paulo passasse a estudar os efeitos da poluição do ar causada pela queima dos canaviais na pré-colheita.

0 primeiro estudo, realizado em Araraquara (SP), encontrou uma associação positiva significativa e dose-dependente entre o número de inalações diárias em serviços de saúde e a concentração de material particulado gerado pela queima da palha da cana-de-açúcar. ${ }^{(37)}$

Outro estudo, realizado na cidade de Piracicaba (SP), confirmou que a queima dos canaviais na pré-colheita leva ao aumento da poluição atmosférica na região. A média anual de $\mathrm{PM}_{10}$ foi de 56 $\mu \mathrm{g} / \mathrm{m}^{3}$, a mesma da cidade de São Paulo (SP) no ano de 1997, com variações entre $88 \mu \mathrm{g} / \mathrm{m}^{3}$, no período da safra, e $29 \mu \mathrm{g} / \mathrm{m}^{3}$ na entressafra. Vale ressaltar que o limite máximo permitido pelo Conselho Nacional de Meio Ambiente é de $50 \mu \mathrm{g} / \mathrm{m}^{3}$. Além disso, observou-se que esta poluição leva a um aumento significativo das internações hospitalares por doenças respiratórias em crianças, adolescentes e idosos. Dessa forma, o estudo concluiu que a queima da palha da cana-de-açúcar é uma importante fonte de poluição atmosférica e que leva ao aumento da morbidade respiratória de forma semelhante à poluição atmosférica produzida por combustíveis fósseis, em grandes centros urbanos. ${ }^{(38-39)}$

\section{EFEITOS CARDIOVASCULARES DA POLUIÇÃO DO AR}

Nos últimos dez anos, estudos experimentais e observacionais têm apresentado evidências consistentes sobre os efeitos da poluição do ar, especialmente do material particulado fino, na morbidade e mortalidade por doenças cardiovasculares (cardíacas, arteriais e cerebrovasculares). Tanto efeitos agudos (aumento de internações e de mortes 
por arritmia, doença isquêmica do miocárdio e cerebral), como crônicos, por exposição em longo prazo (aumento de mortalidade por doenças cerebrovasculares e cardíaca) têm sido relatados. ${ }^{(40-41)}$

0 aumento da poluição do ar tem sido associado ao aumento da viscosidade sangüínea, de marcadores inflamatórios (proteína $\mathrm{C}$ reativa, fibrinogênio) e da progressão da arteriosclerose, a alterações da coagulação, à redução da variabilidade da freqüência cardíaca (indicador de risco para arritmia e morte súbita), à vasoconstricção e ao aumento da pressão arterial, todos fatores de risco para doenças cardiovasculares. ${ }^{(42)}$

Um abrangente e bem controlado estudo prospectivo realizado encontrou risco aumentado de mortalidade relacionada à poluição do ar que de variou de $8 \%$ a $18 \%$, para diversos tipos de doenças cardíacas. Outro estudo realizado em Dublin, Irlanda, ${ }^{(43)}$ revelou que a redução dos níveis de poluentes do ar ocorrida naquela cidade implicou em significativa redução da mortalidade por doenças cardiopulmonares, sugerindo a importância da adoção de medidas preventivas de âmbito coletivo.

Estudos também sugerem que indivíduos idosos e portadores de doenças cardiovasculares prévias, situações cada vez mais freqüentes na sociedade contemporânea, constituem populações mais susceptíveis, reforçando que, além do tabagismo, sedentarismo e dieta, a poluição do ar é um importante fator de risco a ser controlado.

A situação acima exposta não deixa muitas dúvidas sobre qual rumo devemos tomar. Fazem-se necessárias a elaboração e a implementação de um programa de acompanhamento e controle sistemático dos poluentes do ar e seus efeitos sobre a saúde humana, que gerem resultados cientificamente corretos, e que retratem a realidade e auxiliem na promoção de políticas públicas voltadas à melhoria da qualidade de vida dos habitantes da Terra. Assim, é esperado pelos autores deste capítulo que o mesmo seja útil para reforçar a consciência ambiental de nossa sociedade, no sentido de um ambiente mais saudável para nós e nossos descendentes.

\section{REFERÊNCIAS}

1. Elson DM. Atmospheric pollution: a global problem. 2nd ed. Oxford: Blackwell; 1992. p.3.

2. Fircket J. Sur les causes des accidents survenus dans la vallée de la Meuse, lors des brouillards de décembre 1930. Bull Acad R Med Belg. 1931;11:683-741.
3. Shrenk, HH; Heimann, H; Clayton, GD; Gafafer, WM; Wexler, H. Air pollution in Donora, PA: epidemiology of the unusual smog episode of October 1948: preliminary report. Washington: US Public Health Service; 1949. (Public Health Bulletin, 306)

4. Logan WP. Mortality in London fog incident. Lancet. 1953;1(7):336-8.

5. Bascom R, Bromberg PA, Costa DA, Devlin R, Dockery DW, Frampton MW, Lambert W, Samet JM, Speizer FE, Utell M. State Of The Art. Health Effects Of Outdoor Pollution. Am. J. Respir. Crit. Care Med. 1996;153(1):3-50.

6. Saldiva PH, Lichtenfels, AJ, Paiva PS, Barone IA, Martins MA, Massad E, et al. Association between air pollution and mortality due to respiratory diseases in children in Sao Paulo, Brazil: a preliminary report. Environ Res. 1994;65(2):218-25.

7. Saldiva PH, Pope CA 3rd, Schwartz J, Dockery DW, Lichtenfels AJ, Salge JM, et al. Air pollution and mortality in elderly people: a time-series study in Sao Paulo, Brazil. Arch Environ Health. 1995;50(2):159-63.

8. Brunekreef B, Holgate, ST. Air pollution and health. Lancet. 2002;360(9341):1233-42.

9. Schwartz J, Dockery DW. Increased mortality in Philadelphia associated with daily air pollution concentrations. Am Rev Respir Dis. 1992;145(3):600-4.

10. Atkinson RW, Anderson HR, Sunyer J, Ayres J, Baccini M, Vonk JM, et al. Acute effects of particulate air pollution on respiratory admissions: results from APHEA 2 project. Air Pollution and Health: a European Approach. Am J Respir Crit Care Med. 2001;164(10 Pt 1):1860-6.

11. Zanobetti A, Schwartz J, Dockery DW. Airborne particles are a risk factor for hospital admissions for heart and lung disease. Environ Health Perspect. 2000;108(11):1071-7. Comment in: Environ Health Perspect. 2000;108(11):A520-1.

12. Braga AL, Zanobetti A, Schwartz J. The lag structure between particulate air pollution and respiratory and cardiovascular deaths in 10 US cities. J Occup Environ Med. 2001;43(11):927-33.

13. Miraglia, SG El Khouri. Análise do impacto do consumo de diferentes combustíveis na incidência de mortalidade por doenças respiratórias no Município de São Paulo [dissertação]. São Paulo: Escola Politécnica, Universidade de São Paulo; 1997.

14. Lin CA, Martins MA, Farhat SC, Pope CA 3rd, Conceicao $\mathrm{GM}$, Anastacio VM, et al. Air pollution and respiratory illness of children in Sao Paulo, Brazil. Paediatr Perinat Epidemiol. 1999;13(4):475-88.

15. Braga ALF, Conceição GMS, Pereira LAA, Kishi H, Pereira JCR, Andrade MF, et al. Air pollution and pediatric respiratory hospital admissions in Sao Paulo, Brazil. J Environ Med. 1999;1:95-102.

16. Braga AL, Saldiva PH, Pereira LA, Menezes JJ, Conceicao $\mathrm{GM}$, Lin CA, et al.Health effects of air pollution exposure on children and adolescents in Sao Paulo, Brazil. Pediatr Pulmonol. 2001;31(2):106-13.

17. Martins LC, Latorre Mdo R, Saldiva PH, Braga AL. Air pollution and emergency room visits due to chronic lower respiratory diseases in the elderly: an ecological time-series study in Sao Paulo, Brazil. J Occup Environ Med. 2002;44(7):622-7.

18. Ackermann-Liebrich U, Leuenberger P, Schwartz J, Schindler C, Monn C, Bolognini G, et al. Lung function and long term exposure to air pollutants in Switzerland. Study on 
Repercussões clínicas da exposição à poluição atmosférica

Air Pollution and lung Diseases in Adults (SAPALDIA) Team. Am J Respir Crit Care Med. 1997;155(1):122-9.

19. Dockery DW, Cunningham J, Damokosh Al, Neas LM, Spengler JD, Koutrakis P, et al. Health effects of acid aerosols on North American children: respiratory symptoms. Environ Health Perspect. 1996;104(5):500-5.

20. Worldwide variation in prevalence of symptoms of asthma, allergic rhinoconjunctivitis, and atopic eczema: ISAAC. The International Study of Asthma and Allergies in Childhood (ISAAC) Steering Committee. Lancet. 1998;351(9111):122532.Comment in: Lancet. 1998;351(9111):1220-1. Lancet. 2001;357(9252):313-4.

21. McDonnell WF, Abbey DE, Nishino N, Lebowitz MD. Long-term ambient ozone concentration and the incidence of asthma in nonsmoking adults: the AHSMOG Study. Environ Res. 1999;80(2 Pt 1):110-21.

22. McConnell R, Berhane K, Gilliland F, London SJ, Islam T, Gauderman WJ, et al. Asthma in exercising children exposed to ozone: a cohort study. Lancet. 2002;359(9304): 386-91. Erratum in: Lancet. 2002;359(9309):896. Erratum in: Lancet. 2002;359(9309):896. Comment in: Clin J Sport Med. 2003;13(1):62-3; Lancet. 2002 ;360(9330):411.

23. Pope CA 3rd, Burnett RT, Thun MJ, Calle EE, Krewski D, Ito $\mathrm{K}$, et al.Lung cancer, cardiopulmonary mortality, and long-term exposure to fine particulate air pollution. JAMA. 2002;287(9):1132-41. Comment in: JAMA. 2002;288(7):830; author reply 830 .

24. Hoek G, Brunekreef B, Goldbohm S, Fischer P, van den Brandt PA. Association between mortality and indicators of traffic-related air pollution in the Netherlands: a cohort study. Lancet. 2002;360(9341):1203-9. Comment in: Lancet. 2002;360(9341):1184-5. Lancet. 2003;361(9355): 430; author reply 430.

25. American Thoracic Society. What constitutes an adverse health effect of air pollution? Official statement of the American Thoracic Society. Am J Respir Crit Care Med. 2000;161(2 Pt 1):665-73.

26. Arbex MA, Cançado JED, Pereira LAA, Braga ALF, Saldiva PHN. Queima de biomassa e suas repercussões sobre a saúde. J Bras Pneumol. 2004;30(2):158-75.

27. Albalak R, Frisancho AR, Keeler GJ. Domestic biomass fuel combustion and chronic bronchitis in two rural Bolivian villages. Thorax. 1999;54(11):1004-8.

28. Anderson HR. Chronic lung disease in the Papua New Guinea highlands. Thorax. 1979;34(5):647-53.

29. Smith KR, Samet, JM, Romieu 1, Bruce N. Indoor air pollution in developing countries and acute lower respiratory infections in children. Thorax. 2000;55(6):518-32.

30. Houtmeyers E, Gosselink R, Gayan-Ramirez G, Decramer M. Regulation of mucociliary clearance in health and disease. Eur Respir J. 1999;13(5):1177-88. Comment in:
Eur Respir J. 1999;13(5):949-50.

31. Hajat S, Haines A, Goubet AS, Atkinson RW, Anderson HR. Association of air pollution with daily GP consultants for asthma and lower respiratory conditions in London. Thorax. 1999;54(7):597-605.

32. Gharaibeh NS. Effects of indoor air pollution on lung function of primary school children in Jordan. Ann Trop Paediatr. 1996;16(2):97-102.

33. Perez-Padilla R, Regalado J, Vedal S, Pare P, Chapela R, Sansores R, et al. Exposure to biomass smoke and chronic airway disease in Mexican women. A case-control study. Am J Respir Crit Care Med. 1996;154(3 Pt 1):701-6.

34. Boy E, Bruce N, Delgado H. Birth weight and exposure to kitchen wood smoke during pregnancy in rural Guatemala. Environ Health Perspect. 2002;110(1):109-14.

35. Brauer M, Hisham-Hashim J. Fires in Indonesia: crisis and reaction. Environ Sci Technol. 1998;32(17):404A-7A.

36. Tan WC, Qiu D, Liam BL, Ng TP, Lee SH, van Eeden SF, et al. The human bone marrow response to acute air pollution caused by forest fires. Am J Respir Crit Care Med. 2000;161(4 Pt 1):1213-7.

37. Arbex MA, Bohm GM, Saldiva PH, Conceicao GM, Pope AC 3rd, Braga AL. Assessment of the effects of sugar cane plantation burning on daily counts of inhalation therapy. J Air Waste Manag Assoc. 2000;50(10):1745-9.

38. Cançado JED, Saldiva PHN, Pereira LAA, Lara LBLS, Artaxo P, Martinelli LA, et al. The Impact of Sugar Cane-Burning Emissions on the Respiratory System of Children and the Elderly. Environ Health Perspect 2006;114:725-729.

39. Dockery DW, Pope CA 3rd, Xu X, Spengler JD, Ware JH, Fay ME, et al. An association between air pollution and mortality in six U.S. cities. N Engl J Med. 1993;329(24):1753-9. Comment in: N Engl J Med. 1993;329(24):1807-8. N Engl J Med. 1994;330(17):1237-8; N Engl J Med. 2004;350(2): 198-9.

40. Dockery DW. Epidemiologic evidence of cardiovascular effects of particulate air pollution. Environ Health Perspect. 2001;109(Suppl 4):483-6.

41. Brook RD, Franklin B, Cascio W, et al. Air Pollution and Cardiovascular Disease. AHA Scientific Statement. Circulation 2004;109:2655-71

42. Pope CA 3rd, Burnett RT, Thurston GD, Thun MJ, Calle EE, Krewski D, et al. Cardiovascular mortality and long-term exposure to particulate air pollution: epidemiological evidence of general pathophysiological pathways of disease. Circulation. 2004;109(1):71-7. Comment in: Circulation. 2004;109(1):5-7.

43. Clancy L, Goodman P, Sinclair H, Dockery DW. Effect of air-pollution control on death rates in Dublin, Ireland: an intervention study. Lancet. 2002;360(9341):1210-4. Comment in: Lancet. 2002;360(9341):1184-5. 